\title{
The effectiveness of infliximab for Kawasaki disease in children: systematic review and meta-analysis
}

\author{
Dan $\mathrm{Li}^{1,2}$, Xiaohui $\mathrm{Li}^{1,2}$, Wenting Dou ${ }^{1}$, Yang Zheng $^{1}$ \\ ${ }^{1}$ Department of Cardiology, Children's Hospital Capital Institute of Pediatrics, Beijing, China; ${ }^{2}$ Graduate School of Peking Union Medical College, \\ Capital Institute of Pediatrics, Beijing, China \\ Contributions: (I) Conception and design: D Li, X Li; (II) Administrative support: X Li; (III) Provision of study materials or patients: D Li; (IV) \\ Collection and assembly of data: All authors; (V) Data analysis and interpretation: D Li, X Li; (VI) Manuscript writing: All authors; (VII) Final \\ approval of manuscript: All authors. \\ Correspondence to: Xiaohui Li, MD, PhD. Children's Hospital Capital Institute of Pediatrics, Graduate School of Peking Union Medical College, \\ No.2 Ya-Bao Road, Chaoyang District, Beijing 100020, China. Email: lxhmaggie@126.com.
}

Background: Kawasaki disease (KD) is a self-limited illness that results in coronary artery aneurysms (CAAs) and threatens children's health and lives. The therapeutic effects of single intravenous immunoglobulin gamma (IVIG) vs. infliximab (IFX) (with or without IVIG) in young children with KD remain unclear. Thus, we made a meta-analysis and systematic review, including all of the studies which have evaluated the effectiveness and safety of IFX and IVIG KD patients.

Methods: The databases of the Cochrane Library, PubMed and Embase websites were searched for articles appearing from inception until December 31, 2020. Clinical studies that compared IFX either as initial therapy plus IVIG or rescue therapy after IVIG (IFX group) failure compared with IVIG treatment alone (IVIG group) in treating KD patients were included.

Results: The meta-analysis included nine studies characterizing 712 patients. The treatment response was significantly higher in the adjunctive IFX therapy group than in the IVIG therapy group [odds ratio (OR) 2.64; 95\% CI: $1.52-4.59 ; \mathrm{P}=0.0005]$. Subgroup analysis, the effect of IFX therapy on treatment response is more effectiveness in the group of the high-risk KD patients than IVIG therapy (OR 6.07; 95\% CI: 2.30-16.04; $\mathrm{P}=0.0003$; random-effects model). Further analysis showed no difference in the improvement of CAAs in short-term follow-up between the two groups. However, adding IFX either as initial therapy or as additional therapy all showed an advantageous effect regarding the $\Delta \mathrm{Z}$ score of the left anterior descending (LAD) (MD $=0.29 ; 95 \%$ CI: $0.27-0.31 ; \mathrm{P}<0.00001)$ and right coronary artery (RCA) (MD =0.24; 95\% CI: 0.22-0.26; $\mathrm{P}<0.00001)$. Further, IFX exhibited significant effect on the treatment response compared with IVIG therapy in the Asian group (OR, 2.84; 95\% CI: 1.51-5.36; P=0.001; random-effects model), and the beneficial effects of IFX were given without increasing the risk of AEs.

Conclusions: This meta-analysis emphasizes the importance of IFX on the treatment response in the high-risk KD patients. IFX may play a role in the Asian KD patients and prevention of progressive CAA, and does not increase the risk of AEs in KD patients.

Keywords: Infliximab; Kawasaki disease (KD); children; meta-analysis

Submitted Dec 30, 2020. Accepted for publication Mar 18, 2021.

doi: $10.21037 /$ tp-20-482

View this article at: http://dx.doi.org/10.21037/tp-20-482 


\section{Introduction}

Kawasaki disease (KD) is an acute vasculitis mainly presents in children and infants of unknown etiology. It has been increasingly reported worldwide since it was first described in 1967 by Tomisaku Kawasaki in Japan (1). KD is a self-limiting illness that mainly affects the mediumsize arteries and results in coronary artery aneurysms (CAAs) in up to $25 \%$ of untreated children. It is now the primary reason of children's acquired heart disease, and the patients with CAAs may carry a high risk of coronary artery complications such as coronary artery dilation, coronary artery aneurysms, thrombogenesis, myocardial infarction, and sudden death (2).

The standard therapy for KD patients is intravenous immunoglobulin gamma (IVIG) and aspirin, which reduces the risk of CAAs from $25 \%$ to $5 \%$ (3). However, studies have shown that $10-38 \%$ of the patients fail to react or develop a recrudescent fever. These patients are characterized as IVIG resistant, are at high risk of developing CAAs, and require additional therapy to interrupt the inflammatory reaction (4). We postulate that KD patients may benefit from more intensive initial therapy (5).

Infliximab (IFX) is a novel chimeric monoclonal antibody that produces anti-inflammatory effects through specific blocking of tumor necrosis factor-alpha (TNF- $\alpha$ ), the first anti-TNF- $\alpha$ monoclonal antibody treatment validated for pediatric patients. It is safe and well-tolerated and has been used to treat other disease such as spondylitis, rheumatoid arthritis, and Crohn's disease (6). The application of IFX in $\mathrm{KD}$ was first reported by Burns et al. (7), and since then, research has demonstrated that IFX plays an active role in $\mathrm{KD}$ as remedial therapy or initial intensive therapy (8). A prior study by Tremoulet et al. (9) showed the use of IFX plus IVIG as initial therapy in KD patients decreased fever duration, inflammation markers, and the $Z$ score of the left anterior descending (LAD) coronary artery. Yamaji et al. (10) reported that TNF- $\alpha$ blockers including IFX and etanercept in 5 RCTs that compared TNF- $\alpha$ blockers to placebo or other drugs in children with KD. However, RCT studies are rigorously designed, whether such results are appropriate for other clinical conditions remains unclear. The present study aims to evaluate the effectiveness of IFX either as initial or additional therapy in all studies not only RCTs, but also observational studies and case-control studies in the KD patients.

The study protocol was registered on the PROSPERO database (ID 143267). This meta-analysis was performed following the guidelines of the Preferred Reporting Items for Systematic Reviews and Meta-analyses (PRISMA) Statement (11) (available at http://dx.doi.org/10.21037/tp20-482).

\section{Methods}

\section{Study selection criteria}

Prospective cohort designs, retrospective observational studies and case-control studies that in comparison IFX (either as initial or rescue therapy) with IVIG treatment for $\mathrm{KD}$ were included. Studies were considered to be eligible for inclusion when meeting the following criteria: (I) the included patients were children under the age of 18 years diagnosed with KD (12); (II) the intervention of the IFX group referred to using adjunctive IFX either as initial or additional therapy; (III) a comparison was made between the IFX group and the IVIG group; (IV) the outcome evaluation included the treatment response of IFX, the effectiveness of IFX either as initial and additional therapy, the incidence rate of CAA, hospital stay, and AEs after treatment.

\section{Literature search strategy and data extraction}

The PubMed, Medline, Embase, Web of Science, and Cochrane library electronic databases inclusive until December 31, 2020 were searched and the words and MESH terms "Kawasaki disease" OR "Kawasaki syndrome" OR "Mucocutaneous Lymph Node Syndrome,", "TNF- $\alpha$ " OR "Tumor Necrosis Factor-alpha" and "Infliximab" in different forms. A manual and electronic search of references from eligible and relevant studies was performed to find additional trials, and only articles written in the English language were considered eligible (Table S1). The titles and abstracts for the articles identified were assessed by two authors (XL and DL) to determine whether they met the inclusion criteria. Reviews, comments or editorials, conference abstracts, case reports, letters, reviews and metaanalyses were rejected from the analysis. Seventeen articles were reassessed by reviewing the full text, and only studies (with or without randomization) comparing outcomes between two groups (an IFX group and IVIG group) were eligible in the final result. Two independent observers (XL and DL) extracted information from each study, and two other authors (WD and YZ) reviewed the data extraction for completeness and accuracy. 


\section{Data collection process}

We based the Cochrane recommendation review (Cochrane Handbook for Systematic Reviews of Interventions, version 5.1.0 (http://handbook.cochrane.org/) and evaluated study quality according to study objectives, study design, study performance, outcome evaluation and effectiveness. Study characteristics (study purpose, study design, inclusion, and exclusion criteria); patient characteristics (race, age, sex and severity of disease); interventions (therapeutic method, doses, and treatment duration); and outcomes (incidence of CAA, treatment response, impact, and adverse events) were extracted by two reviewers (XL and DL) from eligible studies. Data was then cross-checked using RevMan Version 5.4 (The Nordic Cochrane Centre Collaboration, Copenhagen, Denmark). The article selection, data abstraction, computation, calculation, evaluation, and synthesis processes were reviewed by two authors (XL and $\mathrm{DL}$ ), and the other two authors (WD and $\mathrm{YZ}$ ) resolved disagreements through a joint examination of the articles and discussion until reach a consensus.

\section{Case definition}

This meta-analysis investigated randomized controlled trials (RCTs) and non-RCTs comparing IFX and IVIG therapies for KD children. Treatment response refers to the percentage of patients whose fever subsided within $48 \mathrm{~h}$ with IFX or IVIG therapy with the total patients in each group. Treatment resistance was defined as persistent or recrudescent fever (axillary temperature $>37.5^{\circ} \mathrm{C}$ ) at 48 -hour after the completion of IFX or IVIG infusion, regardless of initial treatment or rescue treatment.

The CAA of KD was defined by the $\mathrm{Z}$ score system of the 2017 AHA scientific statement (2) as follows: (I) no involvement: $<2$; (II) dilation: 2 to $<2.5$; or if initially $<2$, a decrease in $Z$ score during follow-up $\geq 1$; (III) small aneurysm: $\geq 2.5$ to $<5$; (IV) medium aneurysm: $\geq 5$ to $<10$, or absolute dimension $<8 \mathrm{~mm}$; (V) large or giant aneurysm: $\geq 10$, or absolute dimension $\geq 8 \mathrm{~mm}$. The $\Delta Z$ score of the left anterior descending artery (LAD) and right coronary artery (RCA) was the difference between the primary $Z$ score and the follow-up $Z$ score.

\section{Risk of bias}

According to the GRADE Working Group, the risk of bias of eligible studies was assessed to assess the risk of bias in studies $(13,14)$. Seven criteria were used to assess the studies' limitation: (I) random sequence generation (selection bias), (II) allocation concealment (selection bias), (III) blinding of participants and personnel (performance bias), (IV) blinding of outcome assessment (detection bias), (V) incomplete outcome data (attrition bias), (VI) selective reporting (reporting bias), (VII) other bias. For each criterion, the risk of bias was categorized as low, unclear, or high, and confidence in the estimates for each outcome in studies was assessed using the GRADE method. Publication bias was assessed by the visual inspection of the funnel plots, and confidence in the estimates was based on three levels; high, moderate, and low.

\section{Statistical analysis}

The odds ratios (ORs) were used to estimate the effect and 95\% CIs for dichotomous outcomes and mean difference for continuous outcomes. The random-effects model was used to evaluate the effect of our meta-analysis on the intrinsic differences of study design. Heterogeneity among studies was calculated by Q test and estimated by the $\mathrm{I}^{2}$ statistic (15) and interpreted using the Cochrane Collaboration thresholds. Sensitivity analysis was made to test the stability of the overall results through eliminating individual studies in the presence of significant heterogeneity. Continuous outcome measurements were reported as median and range, and the mean and standard variance was estimated using a simulation formula reported by Hozo et al. (16). Statistical analyses were performed with RevMan, all $\mathrm{P}$ values were 2-tailed, and the statistical significance was 0.05 .

\section{Results}

In our study, a total of 440 relevant articles were searched through the preliminary search. With the further assessment of the remaining 18 relevant studies (Table S2), eight noncomparative studies were excluded, and nine fulltext studies were enrolled in the meta-analysis resulting in 712 children who met the study criteria and were included in this meta-analysis (7-9,17-22). The PRISMA study selection flow diagram is illustrated in (Figure 1). A total of nine randomized and nonrandomized studies were included in the risk of bias assessment, and data were extracted according to each domain (Figure 2). Their relevant ethics committees approved the included studies. Methodological quality assessment of included studies is showed in the Table S3. 


Records identified through Cochrane Library $(n=7)$ $(n=124)$ $(n=309)$

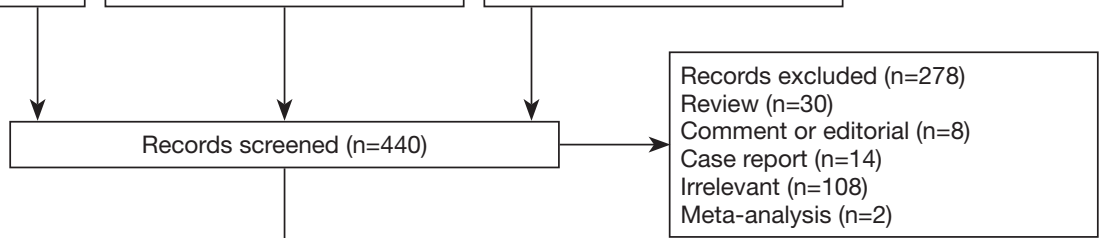

Meta-analysis $(n=2)$

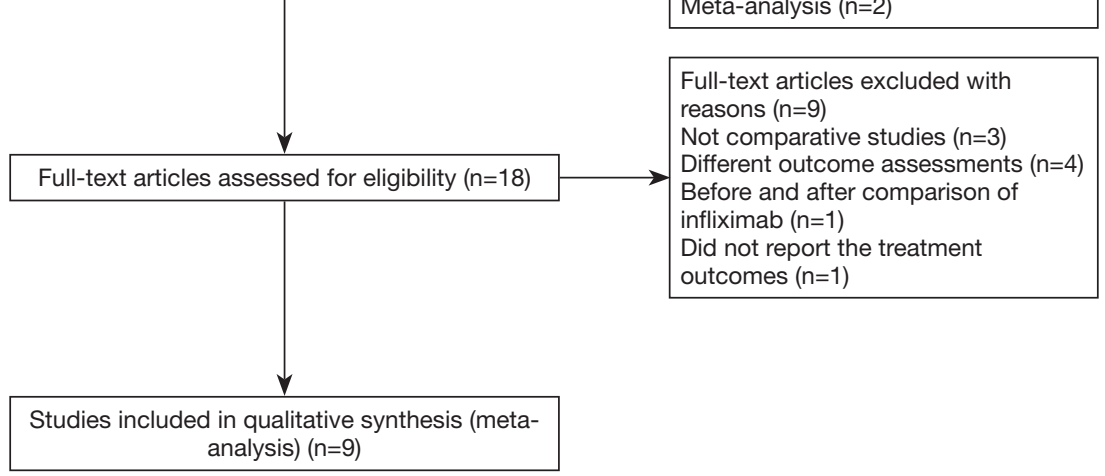

Figure 1 Study selection flow diagram.

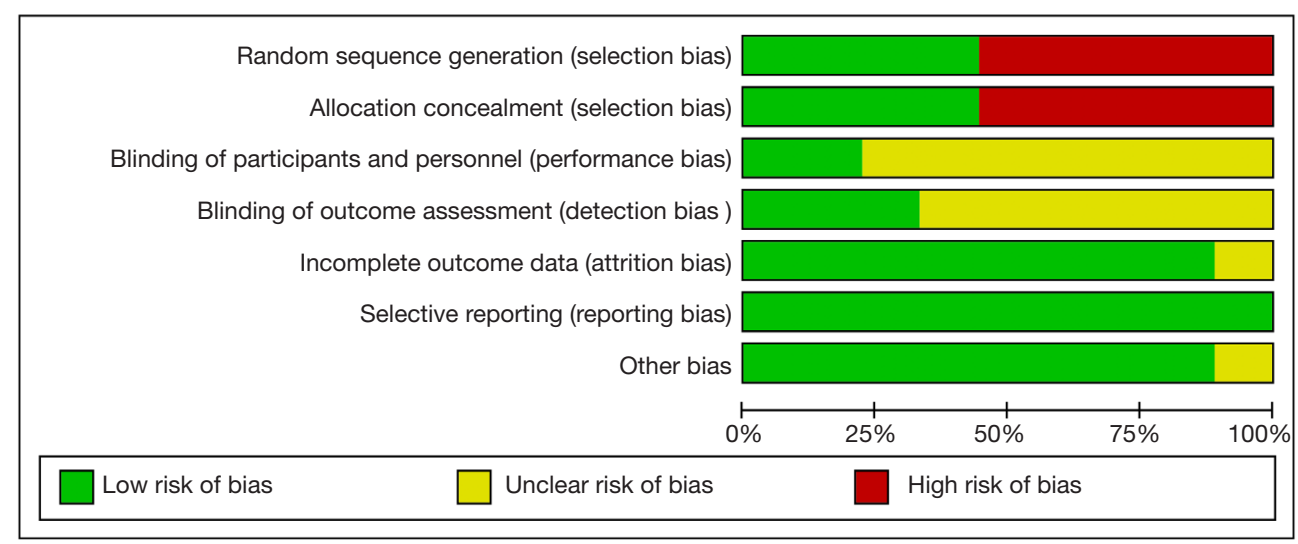

Figure 2 Risk-of-bias graph: authors' judgement of each risk-of-bias item showed as percentages of all studies.

\section{Study characteristics}

The clinical profiles and baseline characteristics of the nine studies are presented in Table 1. The studies were published in English until December 31, 2020 and included four RCTs $(7,9,17,22)$ and five non-RCT $(8,18-21)$ trials. This study involved 712 cases in total (305 in the IFX group and 407 in the IVIG group). The type of studies, the sample size, sex, mean age, the severity of illness, and hospital stay, are also summarized in Table 1. The doses of IFX and IVIG, CAA incidence, and the AEs are detailed in Table 2.

\section{Principal outcome: the overall effectiveness of IFX therapy in all studies on treatment response}

Studies included in the meta-analysis for the overall effectiveness (IFX either as initial or additional therapy) on KD patients' treatment response are shown in Figure 3. We found that the IFX group had a higher treatment response rate (OR, 2.64; 95\% CI: 1.52-4.59; $\mathrm{P}=0.0005$; randomeffects model) compared with the IVIG group. While there were 256 responders of 305 total patients in the IFX group, and the treatment response rate was $84 \%$, there were 285 
Table 1 Baseline characteristics of included studies

\begin{tabular}{|c|c|c|c|c|c|c|c|c|}
\hline Source, year & IFX & $\begin{array}{l}\text { Study } \\
\text { design }\end{array}$ & $\begin{array}{l}\mathrm{CAA} \text { and } \\
\mathrm{CAL}\end{array}$ & Sample size & $\begin{array}{c}\text { Female } \\
\text { patients (\%) }\end{array}$ & $\begin{array}{l}\text { Mean age } \\
\text { (months) }\end{array}$ & Illness severity & Hospital stay (d) \\
\hline $\begin{array}{l}\text { Han et al. (17), } \\
2018\end{array}$ & Initial treatment & RCT & Included & $\begin{array}{l}\text { IFX + IVIG: } 77, \\
\text { IVIG: } 77\end{array}$ & $43(55.8 \%)$ & 25.2 & $\mathrm{KD}$ with $\mathrm{CAA}$ & $\begin{array}{l}\text { Mean (SD): } \\
8.0(2.0)\end{array}$ \\
\hline $\begin{array}{l}\text { Jone et al. (18), } \\
2018\end{array}$ & Initial treatment & Non-RCT & Included & $\begin{array}{l}\text { IFX+IVIG: } 35, \\
\text { IVIG: } 34\end{array}$ & $9(25.7 \%)$ & 25.2 & $\mathrm{KD}$ with $\mathrm{CAL}$ & $\begin{array}{c}\text { Median (range): } \\
3.90(2.30 \text { to } 5.50)\end{array}$ \\
\hline $\begin{array}{l}\text { Youn et al. (20), } \\
2016\end{array}$ & $\begin{array}{l}\text { Additional } \\
\text { treatment }\end{array}$ & Non-RCT & Included & IFX: 11, IVIG: 32 & $15(35 \%)$ & $3.0-156$ & Refractory KD & $\begin{array}{l}\text { Median (range): } \\
8 \text { (7 to } 9)\end{array}$ \\
\hline $\begin{array}{l}\text { Tremoulet } \\
\text { et al. (9), } 2014\end{array}$ & Initial treatment & RCT & Included & $\begin{array}{l}\text { IFX+IVIG: } 97, \\
\text { IVIG: } 98\end{array}$ & 37 (38.8\%) & 33.25 & $\begin{array}{l}\text { Persistent fever } \\
\text { and KD with CAA }\end{array}$ & $\begin{array}{l}\text { Median (range): } \\
3 \text { (4 to } 7)\end{array}$ \\
\hline $\begin{array}{l}\text { Burns et al. (7), } \\
2008\end{array}$ & $\begin{array}{l}\text { Additional } \\
\text { treatment }\end{array}$ & RCT & Included & IFX: 12, IVIG: 12 & $4(33 \%)$ & 20 & $\begin{array}{l}\text { IVIG resistance, } \\
\text { KD with CAA }\end{array}$ & $\begin{array}{l}\text { Median (range): } \\
9.5 \text { (7.8 to } 10.8)\end{array}$ \\
\hline $\begin{array}{l}\text { Mori et al. (22), } \\
2017\end{array}$ & Initial treatment & RCT & Included & IFX:16, IVIG:15 & $6(37.5 \%)$ & 30 & $\begin{array}{c}\text { IVIG resistance, } \\
\text { KD with CAA }\end{array}$ & Not reported \\
\hline
\end{tabular}

IFX, infliximab; IVIG, intravenous immunoglobulin; NR, not reported; RCT, randomized controlled trial.

Table 2 The characteristics of treatment and outcome assessments of included studies

\begin{tabular}{|c|c|c|c|c|c|c|}
\hline Source, year & $\begin{array}{l}\text { Use of Aspirin, } \\
\mathrm{mg} / \mathrm{kg} / \mathrm{d}\end{array}$ & $\begin{array}{l}\text { Use of IFX, } \\
\mathrm{mg} / \mathrm{kg} / \mathrm{d}\end{array}$ & $\begin{array}{l}\text { Use of IVIG, } \\
\text { g/kg/d }\end{array}$ & Criteria of CAA & $\begin{array}{l}\text { Incidence of CAA in each } \\
\text { group, } \mathrm{N}(\%)\end{array}$ & $\begin{array}{c}\text { Serious adverse } \\
\text { events (SAEs) [MD] }\end{array}$ \\
\hline Han et al. (17), 2018 & 80 & 5 & 1 & Japanese criteria & $\begin{array}{l}\text { IFX+IVIG: } 77(3 \%) ; \\
\text { IVIG: } 77(4 \%)\end{array}$ & Not reported \\
\hline Jone et al. (18), 2018 & $80-100$ & 5 & 2 & Z score & $\begin{array}{l}\text { IFX+IVIG: } 35(2 \%) \\
\text { IVIG: } 34(2 \%)\end{array}$ & $\begin{array}{l}\text { IFX+IVIG: } 35 \text { [1]; } \\
\text { IVIG:34 [6] }\end{array}$ \\
\hline Youn et al. (20), 2016 & $80-100$ & 5 & 2 & Japanese criteria & $\begin{array}{l}\text { IFX+IVIG: } 11(1 \%) ; \\
\text { IVIG: } 32(4 \%)\end{array}$ & $\begin{array}{l}\text { IFX+IVIG: } 11[1] ; \\
\quad \text { IVIG:32 [5] }\end{array}$ \\
\hline Tremoulet et al. (9), 2014 & $80-100$ & 5 & 2 & $\begin{array}{l}\text { American Heart } \\
\text { Association case } \\
\text { definition (21) }\end{array}$ & $\begin{array}{l}\text { IFX+IVIG: } 96 \text { (9\%); } \\
\text { IVIG: } 97(4 \%)\end{array}$ & $\begin{array}{l}\text { IFX+IVIG: } 98 \text { [23]; } \\
\text { IVIG: } 98 \text { [22] }\end{array}$ \\
\hline Hirono et al. (8), 2009 & 30 & $5-10$ & 2 & Japanese criteria & $\begin{array}{l}\text { IFX+IVIG: } 11(4 \%) \\
\quad \text { IVIG: } 32(10 \%)\end{array}$ & Not reported \\
\hline Burns et al. (7), 2008 & $80-100$ & 5 & 2 & Z score & $\begin{array}{l}\text { IFX+IVIG: } 12(2 \%) \text {; } \\
\quad \text { IVIG: } 12(2 \%)\end{array}$ & Not reported \\
\hline Mori et al. (22), 2017 & Not reported & 5 & $1-2$ & Z score & $\begin{array}{l}\text { IFX+IVIG: } 16(1 \%) ; \\
\quad \text { IVIG: } 15(3 \%)\end{array}$ & $\begin{array}{l}\text { IFX+IVIG: } 16 \text { [0]; } \\
\text { IVIG: } 15 \text { [1] }\end{array}$ \\
\hline
\end{tabular}

CAAs, coronary artery abnormalities; IVIG, intravenous immunoglobulin. 


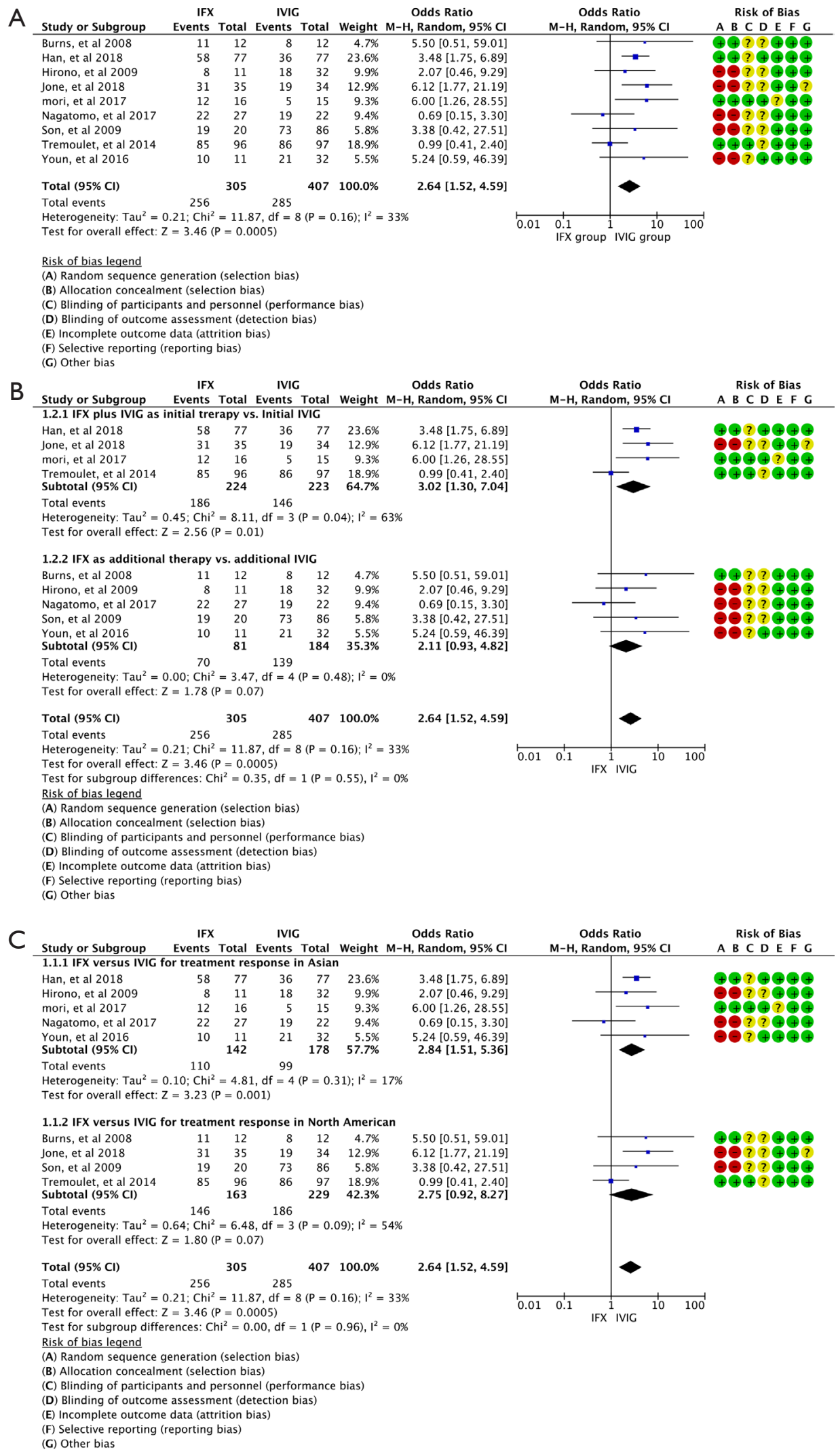




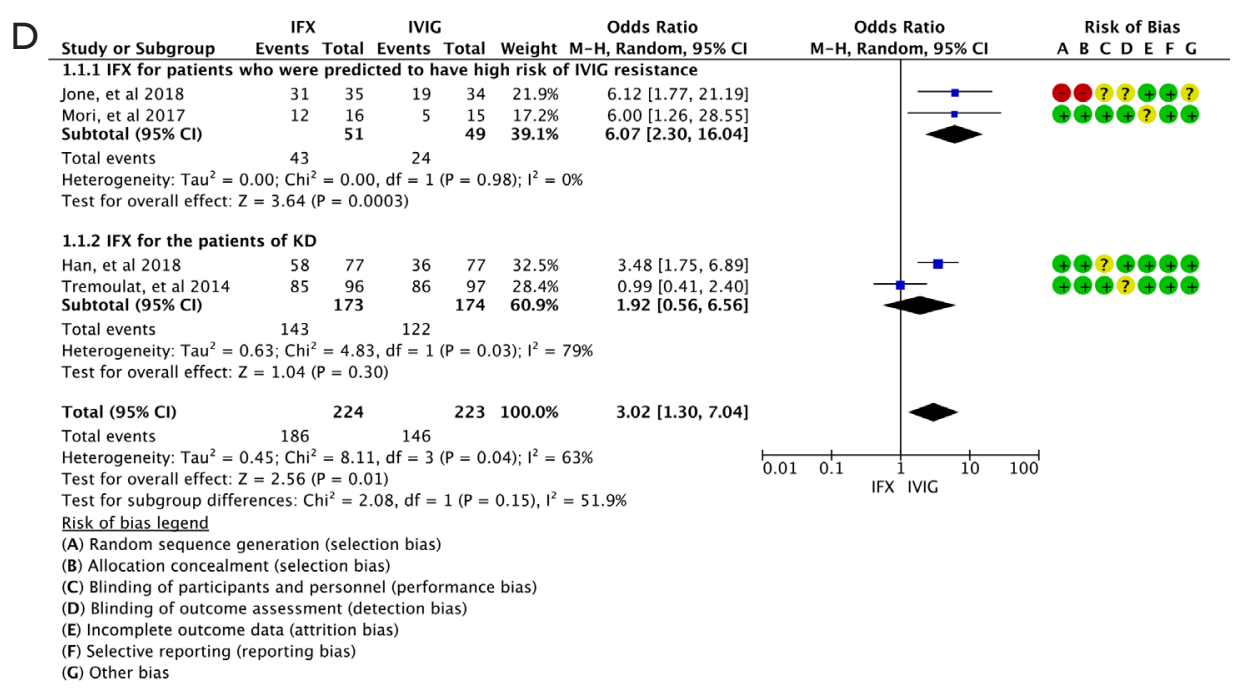

Figure 3 Meta-analysis for the treatment response of KD between the IFX Group and the IVIG Group. (A) The overall effectiveness of IFX therapy in all studies on treatment response. (B) Subgroup 1 shows IFX plus IVIG as initial therapy vs. initial IVIG and subgroup 2 shows IFX as additional therapy after the failure of IVIG treatment $v$ s. additional IVIG. (C) the effectiveness of IFX versus IVIG therapy on treatment response in Asian and North American group; (D) the effectiveness of IFX as initial therapy on treatment response in different risk stratification $\mathrm{KD}$ patients.

responders of 407 total patients in the IVIG group, and the treatment response rate was $70 \%$. There was modest heterogeneity in the included studies $\left(\mathrm{Chi}^{2}=11.87 ; \mathrm{df}=8\right.$; $\mathrm{P}=0.16 ; \mathrm{I}^{2}=33 \%$ ) (Figure 3) and funnel plots in the metaanalyses appeared to be nearly symmetrical.

\section{Subgroup analysis: based on the timing of using IFX (subgroup 1 shows IFX plus IVIG as initial therapy $v$. initial IVIG and subgroup 2 shows IFX as additional therapy after the failure of IVIG treatment $v s$. additional IVIG)}

The effect of IFX either as initial or additional therapy is shown in Figure $3 A, B$. Subset meta-analysis of using IFX plus IVIG as an initial therapy exhibited a significant effect on the treatment response compared with IVIG therapy alone (OR, 3.02; 95\% CI: 1.30-7.04; $\mathrm{P}=0.01$; randomeffects model). However, there was medium heterogeneity (we ran a heterogeneity analysis in the supplement) in this subgroup's meta-analysis $\left(\mathrm{Chi}^{2}=8.11 ; \mathrm{df}=3 ; \mathrm{I}^{2}=63 \%\right.$ ) (Figure $3 A$ ). We also make a subgroup meta-analysis of using IFX for treatment response after remove the study of Tremoulet et al. and we described in the supplementary (Appendix 1, Figure S1). Subgroup analysis for studies using IFX as an adjuvant therapy after failure of IVIG treatment approached clinical significance compared with additional IVIG therapy (OR, 2.11; 95\% CI: 0.93-4.82; $\mathrm{P}=0.07$; random-effects model), and there was no heterogeneity in the subset analysis $\left(\mathrm{Chi}^{2}=3.47 ; \mathrm{df}=4 ; \mathrm{I}^{2}=0 \%\right.$ ) (Figure $3 B$ ).

\section{Subgroup analysis: based on races of using IFX on treatment response (subgroup 1 shows IFX $v s$. IVIG in the Asian group and subgroup 2 shows IFX vs. IVIG in the North American group)}

The effect of IFX either as initial or rescue therapy on the treatment response for KD patients in Asian and North American population is different as shown in Figure 3C. Subset meta-analysis for using IFX either as an initial or rescue therapy strategy exhibited a significant effect on the treatment response compared with IVIG therapy alone in the Asian group (OR, 2.84; 95\% CI: 1.51-5.36; P=0.001; random-effects model) and there was modest heterogeneity in this subgroup meta-analysis $\left(\mathrm{Chi}^{2}=4.81 ; \mathrm{df}=4 ; \mathrm{I}^{2}=17 \%\right)$ (Figure 3C). Subgroup analysis for using IFX in the North American population nearly reached a significant level compared with IVIG therapy on the treatment response (OR, 2.75; 95\% CI: 0.92-8.27; $\mathrm{P}=0.07$; random-effects model), and there was medium heterogeneity in the subset analysis $\left(\mathrm{Chi}^{2}=6.48 ; \mathrm{df}=3 ; \mathrm{I}^{2}=54 \%\right.$ ) (Figure 3 C). 


\section{Subgroup analysis: based on different KD patients} of using IFX on the treatment response (subgroup 1 IFX for KD patients who were predicted high-risk of IVIG resistant and subgroup 2 IFX for the normal KD patients)

From the subgroup analysis of the effect of IFX on the treatment response in the two groups of primary therapy, IFX therapy was more effectiveness in the group of the high-risk KD patients than IVIG therapy alone (OR, 6.07; 95\% CI: 2.30-16.04; $\mathrm{P}=0.0003$; random-effects model), and there was no heterogeneity in this subgroup metaanalysis $\left(\mathrm{Chi}^{2}=0.00 ; \mathrm{df}=1 ; \mathrm{I}^{2}=0 \%\right.$ ) (Figure $3 D$ ). However, IFX showed little advantage in treatment response in the group of normal KD patients compared with IVIG (OR, 3.02; 95\% CI: 1.30-7.04; $\mathrm{P}=0.3$; random-effects model), and there was medium heterogeneity in the subset analysis $\left(\mathrm{Chi}^{2}=4.83 ; \mathrm{df}=1 ; \mathrm{I}^{2}=79 \%\right)$ (Figure $\left.3 D\right)$.

\section{Secondary outcome}

The meta-analysis of KD patients with CAA of KD showed no difference between the IFX group and IVIG group (OR, 1.01; 95\% CI: 0.61-1.66; $\mathrm{P}=0.97$; random-effects model) (Figure $4 A$ ). We have assessed the effect of IFX on KD patients with CAA in rescue therapy and found that it had little significance in rescue therapy compared with IVIG therapy (OR, 0.92; 95\% CI: 0.49-1.74; $\mathrm{P}=0.97$; randomeffects model), and there was no heterogeneity in included studies $\left(\mathrm{Chi}^{2}=4.10 ; \mathrm{df}=8 ; \mathrm{I}^{2}=0 \%\right.$ ) (Figure $\left.4 B\right)$. We also undertook a meta-analysis for the change of $Z$ score $(\Delta Z)$ of the LAD and RCA between IFX and IVIG groups. Pooled analysis showed that adding IFX to standard therapy could increase the decrease rate of the $Z$ score for KD patients. The $\Delta Z$ score of the LAD was \{mean [SD], 1.15 [2] in the IFX group $v s .0 .47$ [1.235] in the IVIG group; mean difference, 0.29; 95\% CI: 0.27-0.31; $\mathrm{P}<0.00001$, randomeffects model\} (Figure 4C) and the RCA \{mean [SD], 0.72 [1.35] in the IFX group vs. 0.39 [1.16] in the IVIG group; mean difference, $0.24 ; 95 \%$ CI: $0.22-0.26$; $<<0.00001$, random-effects model\} (Figure $4 D$ ) was obviously decreased with IFX either as initial therapy or as additional therapy compared with the IVIG alone. There was no heterogeneity in the analysis of $\Delta \mathrm{Z}$ score $(\mathrm{LAD})\left(\mathrm{Chi}^{2}=0.75 ; \mathrm{df}=2 ; \mathrm{I}^{2}=0 \%\right)$ and in the analysis of $\Delta \mathrm{Z}$ score (RCA) $\left(\mathrm{Chi}^{2}=0.08\right.$; $\mathrm{df}=2$; $\left.\mathrm{I}^{2}=0 \%\right)$.
Subgroup analysis: based on race of using IFX on CAA (subgroup 1 shows IFX vs. IVIG in the Asian group and subgroup 2 shows IFX $v s$. IVIG in the North American group)

We also studied the effect of IFX therapy on CAA for KD patients of different races and found that IFX therapy had no clinical significance in either Asian (OR, 0.72; 95\% CI: 0.35-1.49; $\mathrm{P}=0.38$; random-effects model) or North American races (OR, 1.36; 95\% CI: 0.68-2.72; $\mathrm{P}=0.38$; random-effects model) compared with IVIG therapy, and there was no heterogeneity in the analysis of the Asian group $\left(\mathrm{Chi}^{2}=1.31 ; \mathrm{df}=4 ; \mathrm{I}^{2}=0 \%\right)$ and North American group $\left(\mathrm{Chi}^{2}=1.25 ; \mathrm{df}=3 ; \mathrm{I}^{2}=0 \%\right)$ (Figure $\left.4 E\right)$.

\section{Tertiary outcome (meta-analysis for AEs)}

We assessed the AEs in both groups by evaluating clinical manifestations and laboratory testing. Meta-analysis for the rate of AEs showed there was no obvious difference between the two groups $(15.6 \%$ in the IFX group $v$. $14.3 \%$ in the IVIG group; OR, 0.87; $95 \%$ CI: $0.49-1.55$; $\mathrm{P}=0.64$, random-effects model) (Figure 5) and there was no heterogeneity in the analysis $\left(\mathrm{Chi}^{2}=3.99 ; \mathrm{df}=4 ; \mathrm{I}^{2}=0 \%\right)$. Based on study records, almost all of the AEs were transient and easily recoverable, and no deaths were reported. We also make meta-analysis to evaluate the hospital stays, but because of the different medical system and cost of different country, we analyzed this indicator in the supplement (Appendix 1, Figure S2).

\section{Sensitivity analysis and publication bias}

The overall results were not changed after each study was omitted, which confirmed our meta-analysis results (Figure 3). Moreover, we calculated the pooled proportion of studies with moderate-poor quality, and the results were not substantially different. Publication bias was assessed for the outcomes by visual inspection of the funnel plots and no obvious publication bias was detected among the publications that reported IFX effectiveness for $\mathrm{KD}$ patients (Figure 6).

\section{Discussion}

This meta-regression demonstrated that adding IFX 


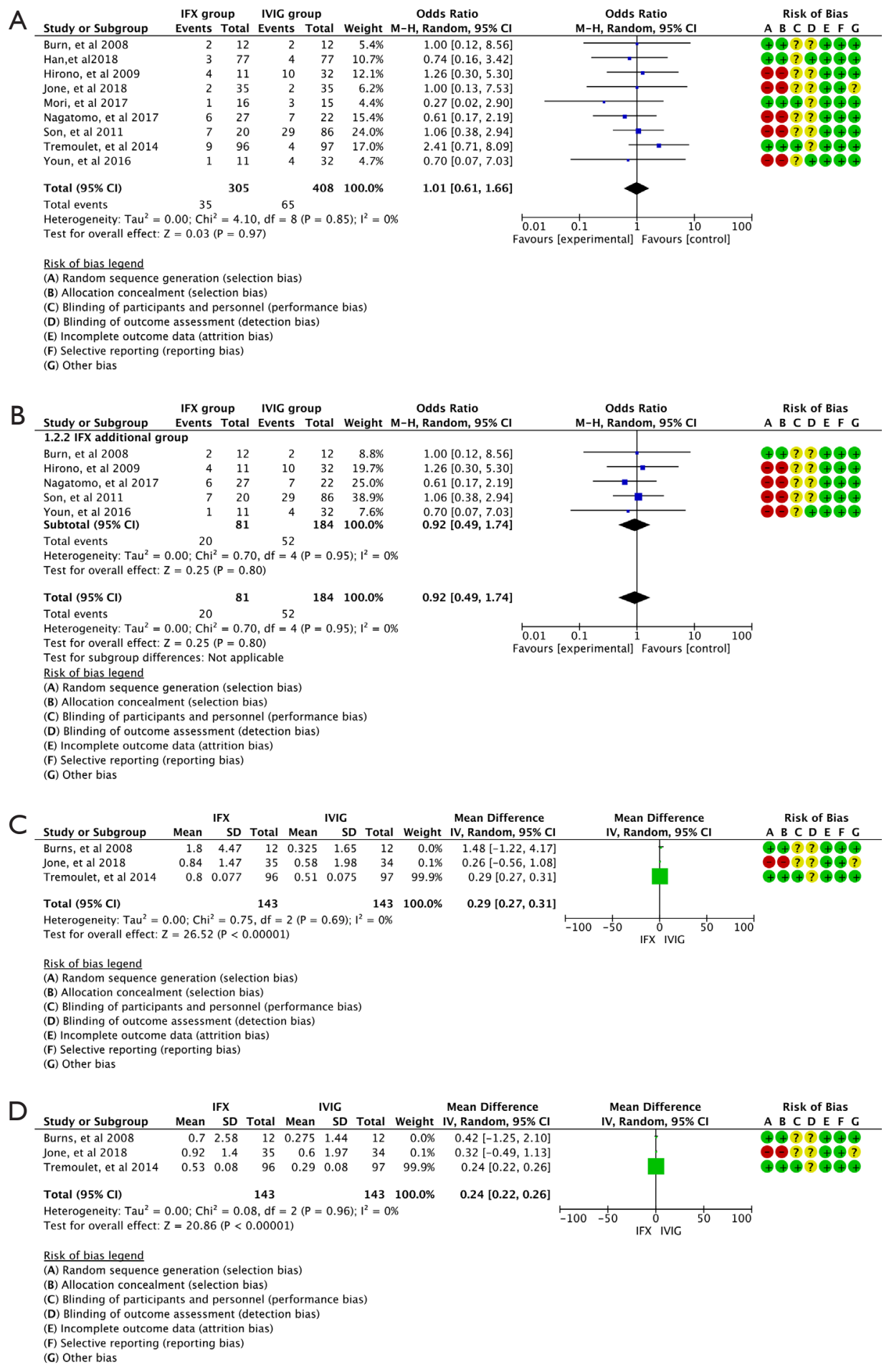




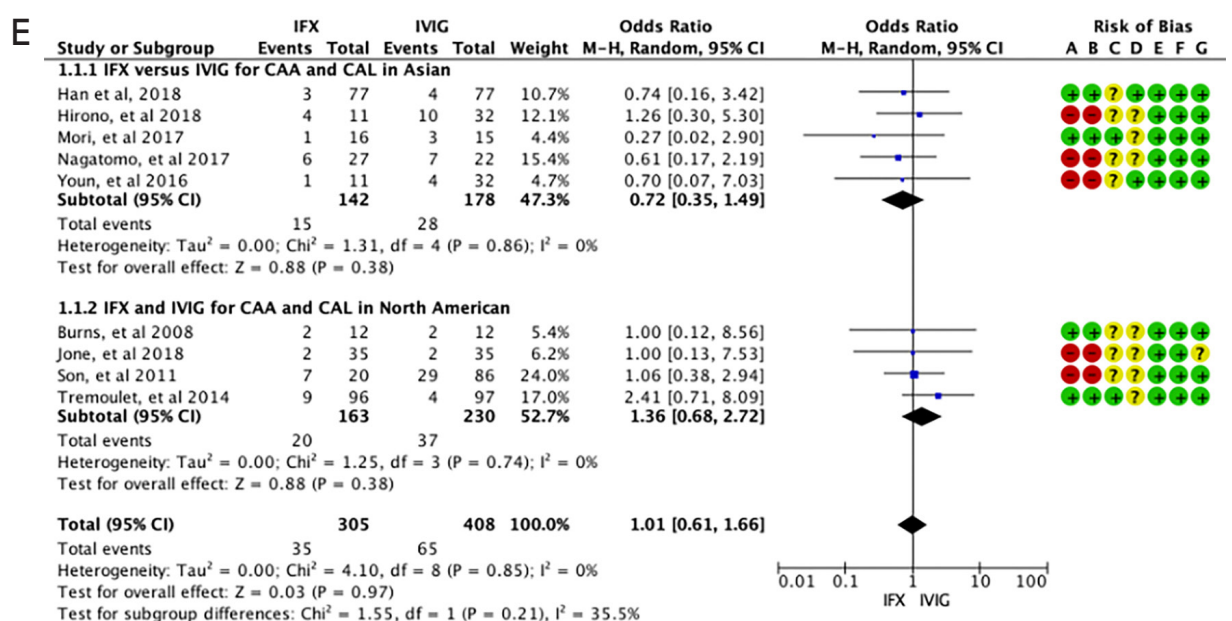

Figure 4 Meta-analysis for the CAA of KD between the IFX Group and the IVIG Group. (A) Meta-analysis for incidence of CAA between the IFX Group and the IVIG group; (B) IFX as rescue therapy versus additional IVIG; (C) meta-analysis for $\Delta \mathrm{Z}$ score (LAD) between the IFX group and the IVIG group; (D) meta-analysis for $\Delta \mathrm{Z}$ score (RCA) between the IFX group and the IVIG group; (E) the effectiveness of IFX versus IVIG therapy on CAA in Asian and North American group.



Figure 5 Meta-analysis of AEs.
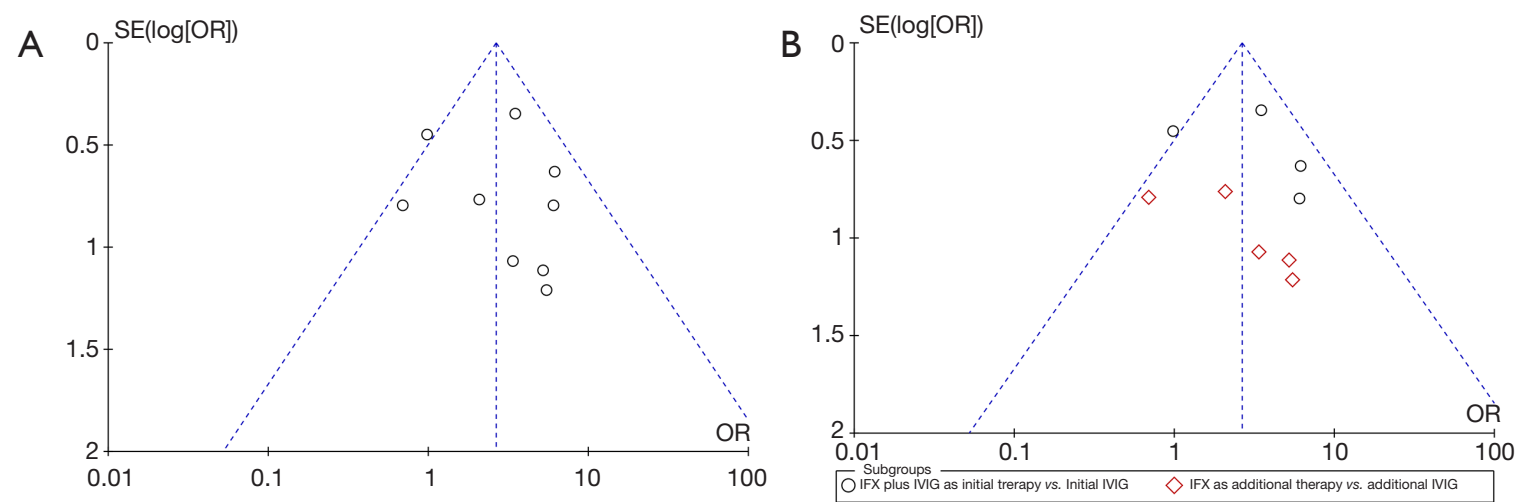

Figure 6 Funnel plots for risk of bias of included studies. 
to traditional IVIG therapy is associated with a higher treatment response for KD patients than the standard treatment. The subgroup showed that KD patients benefit the most from initial IFX with IVIG therapy compared to IVIG alone therapy according to the treatment response. Moreover, the IFX rescue therapy may affect the prevention progressive dilatation of LAD and RCA compared with the IVIG alone. What is more, our analysis showed that the treatment of IFX was more beneficial for the Asian KD patients on treatment response than the North American patients.

Subgroup analysis based on different KD patients showed that IFX as initial therapy was more effectiveness in the group of the high-risk KD patients. However, it is a problem to stratify patients with risk. So far, there are at least 4 different scoring systems, such as Kobayashi score, Egami score, Sano score and Formosa score, for the risk stratification of IVIG resistance have been developed (23-26). These models played useful predictive ability in the early identification of high-risk IVIG resistance patients before the start of treatment.

What is more, the third outcome exhibited that the favorable effects of IFX did not have an increased risk of AEs. This study highlights the importance of IFX on treatment response of KD patients. KD patients benefit greatly from an instant and effective adjunctive IFX therapy.

At present, the dominant view is that the occurrence of $\mathrm{KD}$ may be related to external infection and internal immune dysfunction. Therefore, modulating immune function and reducing inflammation damage is important in its treatment. TNF- $\alpha$ levels are markedly elevated in the acute phase of $\mathrm{KD}$, and children with CAAs have a higher level of TNF- $\alpha$. Increased levels of TNF- $\alpha$ can lead to the aggregation and infiltration of monocytes and neutrophils and induce vascular and coronary artery lesions (27). Theoretically, reducing the level of TNF- $\alpha$ or blocking the binding of TNF- $\alpha$ to its receptor can relieve inflammatory reactions.

The present meta-analysis confirmed the significant role of IFX in KD. Han et al. (17) investigated the treatment effectiveness of traditional IVIG $v s$. combination therapy of IVIG and IFX during the therapeutic process. They found that body temperature, CRP, WBC, and TNF- $\alpha$ in combined therapy patients all showed an earlier and more obvious reduction than those in the IVIG group and that IFX markedly reduced the incidence of CAA in $\mathrm{KD}$ patients compared to traditional IVIG treatment. Furthermore,
Jone et al. (18) suggested that IVIG plus IFX as primary therapy for KD patients with CAA reduced the need of extra second-line treatment, thus decreasing the number of IVIG-resistant patients. Nagatomo et al. (19), showed that the 2-, 4- and 6-year cumulative persistence rate of CAA was $24 \%, 24 \%$ and $24 \%$ in IFX-group, whereas $67 \%, 52 \%$, and $33 \%$ in a non-IFX group, respectively. Therefore, IFX treatment in the long-term follow-up of CAA remains a controversial issue, and more studies and trials are needed to test the importance of IFX on CAA for KD patients.

This study has several significant aspects. First, this study included a list of nine clinical studies characterizing 712 cases, making it the most comprehensive IFX treatment analysis in patients with KD. Second, we found that adding IFX therapy to the conditional therapy was associated with improving the treatment response, prevention of progressive CAA, and without increase of the AEs, which may help reduce the suffering and costs of patients and their families. Furthermore, our subgroup analysis found that IFX exerts a beneficial effect when used as initial treatment to high-risk patients.

\section{Limitations}

There are limitations in the present study. The current evidence of IFX is mainly based on short-term observations, and the long-term studies investigating the safety and efficacy of IFX in patients with CAAs are of great significance and required. Therefore, more studies with long-term follow-up are needed to provide data on the efficacy and safety of IFX. Second, some of the studies also used other drugs such as prednisolone, cyclosporine, and plasmapheresis, there are 9 studies in this paper, of which 5 studies included not only IFX and IVIG, but also prednisolone, cyclosporine, and plasmapheresis. These drugs were used for the IVIG-resistance after IFX or IVIG treatment, and these drugs considered as confounding factors of analysis the efficacy of IFX, which is effective for the IVIG-resistance KD patients, might influence the results.

\section{Conclusions}

In summary, this systematic review and meta-analysis highlight the importance of IFX for KD patients. KD patients benefit greatly from an instant and potent additional IFX therapy to improve the treatment response and prevent coronary artery progressive abnormity. 


\section{Acknowledgments}

Thanks to Professor Lin Zeng for her guidance in the metaanalysis and overall contribution.

Funding: The Beijing Hospital Administration "Peak Climbing" Talents Program (grant number DFL20181301) and the Key Project of Capital Clinical Characteristic Application Research (grant number Z181100001718189).

\section{Footnote}

Reporting Checklist: The authors have completed the PRSIMA reporting checklist. Available at http://dx.doi. org/10.21037/tp-20-482

Peer Review File: Available at http://dx.doi.org/10.21037/tp20-482

Conflicts of Interest: All authors have completed the ICMJE uniform disclosure form (available at http://dx.doi. org/10.21037/tp-20-482). The authors have no conflicts of interest to declare.

Ethical Statement: The authors are accountable for all aspects of the work in ensuring that questions related to the accuracy or integrity of any part of the work are appropriately investigated and resolved.

Open Access Statement: This is an Open Access article distributed in accordance with the Creative Commons Attribution-NonCommercial-NoDerivs 4.0 International License (CC BY-NC-ND 4.0), which permits the noncommercial replication and distribution of the article with the strict proviso that no changes or edits are made and the original work is properly cited (including links to both the formal publication through the relevant DOI and the license). See: https://creativecommons.org/licenses/by-nc$\mathrm{nd} / 4.0 /$.

\section{References}

1. Uehara R, Belay ED. Epidemiology of Kawasaki disease in Asia, Europe, and the United States. J Epidemiol 2012;22:79-85.

2. McCrindle BW, Rowley AH, Newburger JW, et al. Diagnosis, Treatment, and Long-Term Management of Kawasaki Disease: A Scientific Statement for Health Professionals from the American Heart Association.
Circulation 2017;135:e927-99.

3. Newburger JW, Takahashi M, Beiser AS, et al. A single intravenous infusion of gamma globulin as compared with four infusions in the treatment of acute Kawasaki syndrome. N Engl J Med 1991;324:1633-9.

4. Moffett BS, Syblik D, Denfield S, et al. Epidemiology of immunoglobulin resistant Kawasaki disease: results from a large, national database. Pediatr Cardiol 2015;36:374-8.

5. Dominguez SR, Anderson MS, El-Adawy M, et al. Preventing coronary artery abnormalities: a need for earlier diagnosis and treatment of Kawasaki disease. Pediatr Infect Dis J 2012;31:1217-20.

6. Muta H, Ishii M, Furui J, et al. Risk factors associated with the need for additional intravenous gammaglobulin therapy for Kawasaki disease. Acta Paediatr 2006;95:189-93.

7. Burns JC, Best BM, Mejias A, et al. Infliximab treatment of intravenous immunoglobulin-resistant Kawasaki disease. J Pediatr 2008;153:833-8.

8. Hirono K, Kemmotsu Y, Wittkowski H, et al. Infliximab reduces the cytokine-mediated inflammation but does not suppress cellular infiltration of the vessel wall in refractory Kawasaki disease. Pediatr Res 2009;65:696-701.

9. Tremoulet AH, Jain S, Jaggi P, et al. Infliximab for intensification of primary therapy for Kawasaki disease: a phase 3 randomised, double-blind, placebo-controlled trial. Lancet 2014;383:1731-8.

10. Yamaji N, Silva Lopes Kd, Shoda T, et al. TNF- $\alpha$ blockers for the treatment of Kawasaki disease in children. Cochrane Database Syst Rev 2019;8:CD012448.

11. Liberati A, Altman DG, Tetzlaff J, et al. The PRISMA statement for reporting systematic reviews and metaanalyses of studies that evaluate healthcare interventions: explanation and elaboration. BMJ 2009;339:b2700.

12. Research Committee on Kawasaki disease. Report of subcommittee on standardization of diagnostic criteria and re porting of coronary artery lesions in Kawasaki disease. Tokyo, Japan: Ministry of Health and Welfare, 1984.

13. Jadad AR, Moore RA, Carroll D, et al. Assessing the quality of reports of randomized clinical trials: is blinding necessary? Control Clin Trials 1996;17:1-12.

14. Guyatt GH, Oxman AD, Vist GE, et al. GRADE: an emerging consensus on rating quality of evidence and strength of recommendations. BMJ 2008;336:924-6.

15. Lioger B, Maillot F, Ternant D, et al. Efficacy and Safety of Anti-D Immunoglobulins versus Intravenous Immunoglobulins for Immune Thrombocytopenia in Children: Systematic Review and Meta-analysis of 
Randomized Controlled Trials. J Pediatr 2019;204:225-33.e8.

16. Hozo SP, Djulbegovic B, Hozo I. Estimating the mean and variance from the median, range, and the size of a sample. BMC Med Res Methodol 2005;5:13.

17. Han CL, Zhao SL. Intravenous Immunoglobulin Gamma (IVIG) versus IVIG Plus Infliximab in Young Children with Kawasaki Disease. Med Sci Monit 2018;24:7264-70.

18. Jone PN, Anderson MS, Mulvahil MJ, et al. Infliximab Plus Intravenous Immunoglobulin (IVIG) Versus IVIG Alone as Initial Therapy in Children with Kawasaki Disease Presenting with Coronary Artery Lesions: Is Dual Therapy More. Pediatr Infect Dis J 2018;37:976-80.

19. Nagatomo Y, Muneuchi J, Nakashima Y, et al. Effective infliximab therapy for the early regression of coronary artery aneurysm in Kawasaki disease. Int J Cardiol 2018;271:317-21.

20. Youn Y, Kim J, Hong YM, et al. Infliximab as the first retreatment in patients with Kawasaki disease resistant to initial intravenous immunoglobulin. Pediatr Infect Dis J 2016;35:457-9.

21. Son MB, Gauvreau K, Burns JC, et al. Infliximab for Intravenous Immunoglobulin Resistance in

Cite this article as: Li D, Li X, Dou W, Zheng Y. The effectiveness of infliximab for Kawasaki disease in children: systematic review and meta-analysis. Transl Pediatr 2021;10(5):1294-1306. doi: 10.21037/tp-20-482
Kawasaki Disease: A Retrospective Study. J Pediatr 2011;158:644-9.e1.

22. Mori M, Imagawa T, Hara R, et al. Efficacy and limitation of infliximab treatment for children with Kawasaki disease intractable to intravenous immunoglobulin therapy: Report of an open-label case series. J Rheumatol 2012;39:864-7.

23. Kobayashi T, Inoue Y, Takeuchi K, et al. Prediction of intravenous immunoglobulin unresponsiveness in patients with Kawasaki disease. Circulation 2006;113:2606-12.

24. Egami K, Muta H, Ishii M, et al. Prediction of resistance to intravenous immunoglobulin treatment in patients with Kawasaki disease. J Pediatr 2006;149:237-40.

25. Sano T, Kurotobi S, Matsuzaki K, et al. Prediction of nonresponsiveness to standard high-dose gamma-globulin therapy in patients with acute Kawasaki disease before starting initial treatment. Eur J Pediatr 2007;166:131-7.

26. Lin MT, Chang CH, Sun LC, et al. Risk factors and derived formosa score for intravenous immunoglobulin unresponsiveness in Taiwanese children with Kawasaki disease. J Formos Med Assoc 2016;115:350-5.

27. Song MS, Lee SB, Sohn S, et al. Infliximab treatment for refractory Kawasaki disease in Korean children. Korean Circ J 2010;40:334-8. 